\title{
Existence of homoclinic solutions for a class of second order $p$-Laplacian systems with impulsive effects
}

\section{Li Li and Kai Chen*}

\section{"Correspondence:}

chenkai8988@163.com

Faculty of Science, Guilin University

of Aerospace Technology, Guilin,

Guangxi 541004, P.R. China

\begin{abstract}
This paper is concerned with the existence of homoclinic solutions for a class of second order $p$-Laplacian systems with impulsive effects. A new result is obtained under more relaxed conditions by using the mountain pass theorem, a weak convergence argument, and a weak version of Lieb's lemma.
\end{abstract}

MSC: $34 \mathrm{C} 37 ; 35 \mathrm{~A} 15 ; 37 \mathrm{~J} 45 ; 47 \mathrm{~J} 30$

Keywords: existence; homoclinic solutions; variational methods; critical point; impulsive effects

\section{Introduction}

Consider homoclinic solutions of the following problem:

$$
\left\{\begin{array}{l}
\frac{d}{d t}\left(|\dot{u}(t)|^{p-2} \dot{u}(t)\right)-a(t)|u(t)|^{p-2} u(t)+\nabla V(t, u(t))=0, \quad \text { a.e. } t \in\left(t_{j}, t_{j+1}\right), j \in \mathbb{Z}, \\
\Delta\left(\left|\dot{u}\left(t_{j}\right)\right|^{p-2} \dot{u}\left(t_{j}\right)\right)=\left|\dot{u}\left(t_{j}^{+}\right)\right|^{p-2} \dot{u}\left(t_{j}^{+}\right)-\left|\dot{u}\left(t_{j}^{-}\right)\right|^{p-2} \dot{u}\left(t_{j}^{-}\right)=I\left(u\left(t_{j}\right)\right), \quad j \in \mathbb{Z},
\end{array}\right.
$$

where $p \in(1,+\infty), V: \mathbb{R} \times \mathbb{R} \rightarrow \mathbb{R}$ is of class $C^{1}, \nabla V(t, u(t))=\frac{\partial V(t, u(t))}{\partial u}, I: \mathbb{R} \rightarrow \mathbb{R}, a \in$ $C(\mathbb{R},(0,+\infty))$, and $a(t) \rightarrow+\infty$ as $|t| \rightarrow+\infty$. $\mathbb{Z}$ denotes the sets of integers, and $t_{j}(j \in \mathbb{Z})$ are impulsive points. Moreover, there exist a positive integer $m$ and a positive constant $T$ such that $0<t_{0}<t_{1}<\cdots<t_{m-1}<T, t_{l+k m}=t_{l}+k T, \forall k \in \mathbb{Z}, l=0,1, \ldots, m-1 . \dot{u}\left(t_{j}^{+}\right)=$ $\lim _{h \rightarrow 0^{+}} \dot{u}\left(t_{j}+h\right)$ and $\dot{u}\left(t_{j}^{-}\right)=\lim _{h \rightarrow 0^{-}} \dot{u}\left(t_{j}-h\right)$ represent the right and left limits of $\dot{u}(t)$ at $t=t_{j}$, respectively.

When $a(t) \equiv 0$ and $p=2$, problem (1.1) becomes the following problem:

$$
\left\{\begin{array}{l}
\ddot{u}(t)+\nabla V(t, u(t))=0, \quad \text { a.e. } t \in\left(t_{j}, t_{j+1}\right), j \in \mathbb{Z} \\
\Delta \dot{u}\left(t_{j}\right)=\dot{u}\left(t_{j}^{+}\right)-\dot{u}\left(t_{j}^{-}\right)=I\left(u\left(t_{j}\right)\right), \quad j \in \mathbb{Z} .
\end{array}\right.
$$

By using the mountain pass theorem, a weak convergence argument, and a weak version of Lieb's methods, Fang and Duan [1] investigated homoclinic solutions of problem (1.2) and obtained the following main result.

Theorem A [1] Assume that the following conditions hold:

(V1) there exists a positive number $T$ such that

$$
\nabla V(t+T, x)=\nabla V(t, x), \quad V(t+T, x)=V(t, x), \quad \forall(t, x) \in \mathbb{R}^{2} ;
$$


(V2) $\lim _{x \rightarrow 0} \frac{\nabla V(t, x)}{x}=0$ uniformly for $t \in \mathbb{R}$;

(V3) there exists a constant $\mu>2$ such that

$$
\mu V(t, x) \leq(\nabla V(t, x), x), \quad \forall(t, x) \in \mathbb{R} \times \mathbb{R} \backslash\{0\}
$$

(V4) there exist constants $b_{0}>0$ and $b>0$ such that

$$
\begin{aligned}
& V(t, x) \geq b_{0}|x|^{\mu}, \quad \forall t \in \mathbb{R},|x| \geq 1 \\
& V(t, x) \leq b|x|^{\mu}, \quad \forall t \in \mathbb{R},|x| \leq 1
\end{aligned}
$$

(I) there exists a constant $b_{1}$ with $0<b_{1}<\frac{\mu-2}{(\mu+2) m T}$ such that

$$
|I(x)| \leq b_{1}|x|, \quad 2 \int_{0}^{x} I(t) d t-I(x) x \leq 0 .
$$

Then problem (1.2) possesses a nontrivial weak homoclinic orbit.

For $I \neq 0$, problem (1.1) involves impulsive effects. It is well known that impulsive differential equations are used in various fields of science and technology, for example, many biological phenomena involving thresholds, bursting rhythm models in medicine and biology, optimal control models in economics, pharmacokinetics, and frequency modulated systems, and so on. For more details of impulsive differential equations, we refer the readers to the books $[2,3]$.

Recently, the existence and multiplicity of solutions for impulsive differential equations via variational methods have been investigated by some researchers. See for example [417] and references therein. However, there are few papers [1, 18-20] concerning homoclinic solutions of impulsive differential equations by variational methods. So it is a novel method to employ variational methods to investigate the existence of homoclinic solutions for impulsive differential equations.

Motivated by the above papers, we will establish a new result for (1.1).

Let

$$
\begin{gathered}
E=\left\{u \in W^{1, p}(\mathbb{R}, \mathbb{R}) \mid \int_{\mathbb{R}}\left[|\dot{u}(t)|^{p}+a(t)|u(t)|^{p}\right] d t<+\infty,\right. \\
\left.\left\{u\left(t_{j}\right)\right\}_{j=-\infty}^{+\infty} \in l^{2}, u( \pm \infty)=0, u(k T)=0, k \in \mathbb{Z}\right\},
\end{gathered}
$$

where $l^{2}$ denotes the space of sequences whose second powers are summable on $\mathbb{Z}$, that is,

$$
\sum_{j \in \mathbb{Z}}\left|a_{j}\right|^{2}<+\infty, \quad \forall a=\left\{a_{j}\right\}_{j=-\infty}^{+\infty} \in l^{2} .
$$

The space $l^{2}$ is equipped with the following norm:

$$
\|a\|_{l^{2}}=\left(\sum_{j \in \mathbb{Z}}\left|a_{j}\right|^{2}\right)^{1 / 2}
$$


Similar to [1], we can check that $E$ is a Banach space with the norm given by

$$
\|u\|=\left(\int_{\mathbb{R}}\left[|\dot{u}(t)|^{p}+a(t)|u(t)|^{p}\right] d t\right)^{1 / p} .
$$

It is obvious that

$$
E \subset L^{p_{1}}(\mathbb{R})
$$

with the embedding being continuous. Here $L^{p_{1}}(\mathbb{R})\left(p_{1} \in[p,+\infty]\right)$ denotes the Banach spaces of functions on $\mathbb{R}$ with values in $\mathbb{R}$ under the norm

$$
\|u\|_{L^{p_{1}}}=\left\{\int_{\mathbb{R}}|u(t)|^{p_{1}} d t\right\}^{1 / p_{1}} .
$$

Here and in subsequence, $($,$) and |\cdot|$ denote the inner product and norm in $\mathbb{R}$, respectively. $C_{i}(i=1,2, \ldots)$ denote different positive constants. Now, we state our main result.

Theorem 1.1 Suppose that a, I, and V satisfy (V1) and the following conditions:

(A) $\quad a \in C(\mathbb{R},(0,+\infty)), a(t+T)=a(t)$, and $a(t) \rightarrow+\infty$ as $|t| \rightarrow+\infty$;

$(\mathrm{V} 2)^{\prime} V(t, x)=V_{1}(t, x)-V_{2}(t, x), V_{1}, V_{2} \in C^{1}(\mathbb{R} \times \mathbb{R}, \mathbb{R})$, and there exists a constant $R>0$ such that

$$
|\nabla V(t, x)|=o\left(|x|^{p-1}\right) \quad \text { as } x \rightarrow 0 \text { uniformly in } t \in \mathbb{R}
$$

(V3)' there is a constant $\mu>p$ such that

$$
0<\mu V_{1}(t, x) \leq\left(\nabla V_{1}(t, x), x\right), \quad \forall(t, x) \in \mathbb{R} \times \mathbb{R} \backslash\{0\} ;
$$

(V5) $V_{2}(t, 0)=0$ and there exists a constant $\varrho \in(p, \mu)$ such that

$$
\left(\nabla V_{2}(t, x), x\right) \leq \varrho V_{2}(t, x), \quad \forall(t, x) \in \mathbb{R} \times \mathbb{R}
$$

(I)' $\quad I \in C(\mathbb{R}, \mathbb{R})$, and there exists a constant $c$ with $0<c<\frac{(\mu-p)}{(\mu+p) m T^{p / q}}$ such that

$$
|I(x)| \leq c|x|^{p-1}, \quad p \int_{0}^{x} I(t) d t-I(x) x \leq 0, \quad \forall x, y \in \mathbb{R} .
$$

Then problem (1.1) has a nontrivial homoclinic solution.

\section{Preliminaries}

Lemma 2.1 [21] Let $E$ be a real Banach space and $\varphi \in C^{1}(E, \mathbb{R}), e \in E, r>0$ be such that $\|e\|>r$ and

$$
b:=\inf _{\|y\|=r} \varphi(y)>\varphi(0) \geq \varphi(e) .
$$


Let

$$
\Phi=\{h \in C([0,1], E) \mid h(0)=0, h(1)=e\}, \quad d=\inf _{h \in \Phi} \max _{s \in[0,1]} \varphi(h(s)) .
$$

Then, for each $\varepsilon>0, \delta>0$, there exists $y \in E$ such that

(i) $c-2 \varepsilon \leq \varphi(y) \leq c+2 \varepsilon$;

(ii) $\operatorname{dist}(y, E) \leq 2 \delta$;

(iii) $\left\|\varphi^{\prime}(y)\right\| \leq \frac{8 \varepsilon}{\delta}$.

Lemma 2.2 Assume that (V3)' and (V5) hold. Then for every $(t, x) \in \mathbb{R} \times \mathbb{R}$,

(i) $s^{-\mu} V_{1}(t, s x)$ is nondecreasing on $(0,+\infty)$;

(ii) $s^{-\varrho} V_{2}(t, s x)$ is nonincreasing on $(0,+\infty)$.

The proof of Lemma 2.2 is routine and we omit it. Similar to [21-23], we have the following lemma.

Lemma 2.3 For any $u \in E$, the following inequalities hold:

$$
\begin{aligned}
& \|u\|_{\infty}:=\sup _{t \in \mathbb{R}}|u(t)| \leq\left(\max \left\{(p-1) / 2,1 / 2 a_{0}^{p-1}\right\}\right)^{1 / p}\|u\|:=C_{1}\|u\|, \\
& \sum_{j=-\infty}^{+\infty}\left|u\left(t_{j}\right)\right|^{p} \leq m T^{p / q}\|\dot{u}\|_{L^{p}}^{p},
\end{aligned}
$$

where $C_{1}=\left(\max \left\{(p-1) / 2,1 / 2 a_{2}^{p-1}\right\}\right)^{1 / p}, a_{0}=\min _{t \in \mathbb{R}}\{a(t)\}$ from $(\mathrm{A})$.

The following lemma comes from [1], which is similar to a weak version of Lieb's lemma [24].

Lemma 2.4 [1] If $\left\{u_{n}\right\}$ is bounded in $E$ and $u_{n}$ does not converge to 0 in measure, then there exist a sequence $\left\{x_{n_{k}}\right\} \subset \mathbb{Z}$ and a subsequence $\left\{u_{n_{k}}\right\}$ of $\left\{u_{n}\right\}$ such that $u_{n_{k}}\left(\cdot+x_{n_{k}} T\right) \rightarrow u \neq 0$ in $E$.

The functional $\varphi$ corresponding to (1.1) on $E$ is given by

$$
\begin{aligned}
\varphi(u)= & \int_{\mathbb{R}} \frac{1}{p}\left[|\dot{u}(t)|^{p}+a(t)|u(t)|^{p}\right] d t-\int_{\mathbb{R}} V(t, u(t)) d t \\
& +\sum_{j=-\infty}^{\infty} \int_{0}^{u\left(t_{j}\right)} I(s) d s, \quad u \in E .
\end{aligned}
$$

Lemma 2.5 If (V1), (A), (I)', and (V2)' hold, then $\varphi \in C^{1}(E, \mathbb{R})$ and

$$
\begin{aligned}
\left\langle\varphi^{\prime}(u), v\right\rangle= & \int_{\mathbb{R}}\left[|\dot{u}(t)|^{p-2}(\dot{u}(t), \dot{v}(t))+a(t)|u(t)|^{p-2}(u(t), v(t))\right] d t \\
& -\int_{\mathbb{R}}(\nabla V(t, u(t)), v(t)) d t+\sum_{j=-\infty}^{\infty}\left(I\left(u\left(t_{j}\right)\right), v\left(t_{j}\right)\right) .
\end{aligned}
$$

Furthermore, the critical points of $\varphi$ in E are classical solutions of $(1.1)$ with $u( \pm \infty)=0$. 
Proof Firstly, we show that $\varphi: E \rightarrow \mathbb{R}$. By (V2)', for any given $\varepsilon_{0}>0$, there exists $\gamma_{0}>0$ such that

$$
|\nabla V(t, x)| \leq p \varepsilon_{0} a_{0}|x|^{p-1}, \quad t \in \mathbb{R},|x| \leq \gamma_{0} .
$$

Then, by $V(t, 0)=0$ and (2.3), we have

$$
|V(t, x)|=\left|\int_{0}^{1}(\nabla V(t, s x), x) d s\right| \leq \varepsilon_{0} a_{0}|x|^{p}, \quad \forall t \in \mathbb{R}, x \in \mathbb{R} .
$$

From (2.4), we have

$$
\begin{aligned}
\left|\int_{\mathbb{R}} V(t, u(t)) d t\right| & \leq \int_{\mathbb{R}}|V(t, u(t))| d t \\
& \leq \int_{\mathbb{R}} \varepsilon_{0} a_{0}|u(t)|^{p} d t \\
& \leq \int_{\mathbb{R}} \varepsilon_{0} a(t)|u(t)|^{p} d t \leq \varepsilon_{0}\|u\|^{p}, \quad u \in E .
\end{aligned}
$$

From $(\mathrm{I})^{\prime}$ and Lemma 2.3, we have

$$
\begin{aligned}
\sum_{j=-\infty}^{\infty}\left|\int_{0}^{u\left(t_{j}\right)} I(s) d s\right| & \leq \sum_{j=-\infty}^{\infty} \int_{\min \left\{0, u\left(t_{j}\right)\right\}}^{\max \left\{0, u\left(t_{j}\right)\right\}}|I(s)| d s \\
& \leq \frac{1}{p} \sum_{j=-\infty}^{\infty} c\left|u\left(t_{j}\right)\right|^{p} \leq \frac{c m T^{p / q}}{p}\|\dot{u}\|_{L^{p}}^{p} \leq \frac{c m T^{p / q}}{p}\|u\|^{p} .
\end{aligned}
$$

It follows from (2.1), (2.5), and (2.6) that $\varphi: E \rightarrow \mathbb{R}$. Next we prove that $\varphi \in C^{1}(E, \mathbb{R})$. Rewrite $\varphi$ as follows:

$$
\varphi(u)=\varphi_{1}(u)-\varphi_{2}(u)+\varphi_{3}(u),
$$

where

$$
\begin{aligned}
& \varphi_{1}(u):=\int_{\mathbb{R}} \frac{1}{p}\left[|\dot{u}(t)|^{p}+a(t)|u(t)|^{p}\right] d t, \\
& \varphi_{2}(u):=\int_{\mathbb{R}} V(t, u(t)) d t, \quad \varphi_{3}(u):=\sum_{j=-\infty}^{\infty} \int_{0}^{u\left(t_{j}\right)} I(s) d s .
\end{aligned}
$$

It is easy to check that $\varphi_{1}, \varphi_{3} \in C^{1}(E, \mathbb{R})$ and

$$
\begin{aligned}
& \left\langle\varphi_{1}^{\prime}(u), v\right\rangle=\int_{\mathbb{R}}\left[|\dot{u}(t)|^{p-2}(\dot{u}(t), \dot{v}(t))+a(t)|u(t)|^{p-2}(u(t), v(t))\right] d t, \\
& \left\langle\varphi_{3}^{\prime}(u), v\right\rangle=\sum_{j=-\infty}^{\infty}\left(I\left(u\left(t_{j}\right)\right), v\left(t_{j}\right)\right), \quad \forall u, v \in E .
\end{aligned}
$$

Next we prove that $\varphi_{2} \in C^{1}(E, \mathbb{R})$ and

$$
\left\langle\varphi_{2}^{\prime}(u), v\right\rangle=\int_{\mathbb{R}}(\nabla V(t, u(t)), v(t)) d t, \quad \forall u, v \in E .
$$


By (2.3), we have

$$
\begin{aligned}
\left|\left(\nabla V\left(t, u_{n}(t)\right), v(t)\right)\right| & \leq \varepsilon_{0} p a_{0}\left|u_{n}(t)\right|^{p-1}|v(t)| \\
& \leq 2^{p-2} \varepsilon_{0} p a(t)\left(\left|u_{n}(t)-u(t)\right|^{p-1}+|u(t)|^{p-1}\right)|v(t)| \\
& :=g_{n}(t), \quad t \in \mathbb{R} .
\end{aligned}
$$

Let $u_{n} \rightarrow u$ in $E$, so $u_{n}(t) \rightarrow u(t)$ for almost every $t \in \mathbb{R}$, we have

$$
\lim _{n \rightarrow+\infty} g_{n}(t)=2^{p-2} p \varepsilon_{0} a(t)|u(t)|^{p-1}|v(t)|:=g(t)
$$

and

$$
\begin{aligned}
& \lim _{n \rightarrow+\infty} \int_{\mathbb{R}} g_{n}(t) d t \\
& \quad=\lim _{n \rightarrow+\infty} \int_{\mathbb{R}}\left[2^{p-2} p \varepsilon_{0} a(t)\left(\left|u_{n}(t)-u(t)\right|^{p-1}+|u(t)|^{p-1}\right)|v(t)|\right] d t \\
& =\lim _{n \rightarrow+\infty} \int_{\mathbb{R}} 2^{p-2} p \varepsilon_{0} a(t)\left|u_{n}(t)-u(t)\right|^{p-1} d t+\int_{\mathbb{R}}\left[2^{p-2} p \varepsilon_{0} a(t)|u(t)|^{p-1}|v(t)|\right] d t \\
& =\int_{\mathbb{R}}\left[2^{p-2} p \varepsilon_{0} a(t)|u(t)|^{p-1}|v(t)|\right] d t \\
& :=\int_{\mathbb{R}} g(t) d t<+\infty .
\end{aligned}
$$

Then by (2.7), (2.8), (2.9), and Lebesgue's dominated convergence theorem, we have

$$
\lim _{n \rightarrow+\infty} \int_{\mathbb{R}}\left(\nabla V\left(t, u_{n}(t)\right), v(t)\right) d t=\int_{\mathbb{R}}(\nabla V(t, u(t)), v(t)) d t
$$

Therefore, for any $u, v \in E$ and for any function $\theta: \mathbb{R} \rightarrow(0,1)$, from (2.10), we have

$$
\begin{aligned}
\left\langle\varphi_{2}^{\prime}(u), v\right\rangle & =\lim _{h \rightarrow 0^{+}} \frac{\varphi_{2}(u+h v)-\varphi_{2}(u)}{h} \\
& =\lim _{h \rightarrow 0^{+}} \frac{1}{h} \int_{\mathbb{R}}[V(t, u(t)+h v(t))-V(t, u(t))] d t \\
& =\lim _{h \rightarrow 0^{+}} \int_{\mathbb{R}}(\nabla V(t, u(t)+\theta(t) h v(t)), v(t)) d t \\
& =\int_{\mathbb{R}}(\nabla V(t, u(t)), v(t)) d t, \quad \forall u, v \in E .
\end{aligned}
$$

Now, we prove that $\varphi_{2} \in C^{1}(E, \mathbb{R})$. From $(2.11), u_{n} \rightarrow u$ in $E$ and $V \in C^{1}(\mathbb{R} \times \mathbb{R}, \mathbb{R})$, we have

$$
\begin{aligned}
& \lim _{n \rightarrow+\infty}\left|\left\langle\varphi_{2}^{\prime}\left(u_{n}\right)-\varphi_{2}^{\prime}(u), v\right\rangle\right| \\
& \quad=\lim _{n \rightarrow+\infty}\left|\int_{\mathbb{R}}\left(\nabla V\left(t, u_{n}(t)\right)-\nabla V(t, u(t)), v(t)\right) d t\right| \\
& \quad \leq \lim _{n \rightarrow+\infty} \int_{\mathbb{R}}\left|\nabla V\left(t, u_{n}(t)\right)-\nabla V(t, u(t))\right||v(t)| d t \\
& \quad=\int_{\mathbb{R}} \lim _{n \rightarrow+\infty}\left|\nabla V\left(t, u_{n}(t)\right)-\nabla V(t, u(t))\right||v(t)| d t=0, \quad \forall v \in E .
\end{aligned}
$$


This shows that $\varphi_{2} \in C^{1}(E, \mathbb{R})$. Therefore $\varphi \in C^{1}(E, \mathbb{R})$ and (2.2) holds. Finally, we prove that the critical points of $\varphi$ in $E$ are classical solutions of (1.1) with $u( \pm \infty)=0$. Let $u \in E$ be a critical point of $\varphi$, then for any $v \in C_{0}^{\infty}(\mathbb{R})$, we have

$$
\begin{aligned}
0= & \left\langle\varphi^{\prime}(u), v\right\rangle \\
= & \int_{\mathbb{R}}\left[|\dot{u}(t)|^{p-2}(\dot{u}(t), \dot{v}(t))+a(t)|u(t)|^{p-2}(u(t), v(t))\right] d t \\
& -\int_{\mathbb{R}}(\nabla V(t, u(t)), v(t)) d t+\sum_{j=-\infty}^{\infty}\left(I\left(u\left(t_{j}\right)\right), v\left(t_{j}\right)\right) .
\end{aligned}
$$

Let $v \in C_{0}^{\infty}(\mathbb{R})$ such that $v(t)=0$ for any $t \in\left(-\infty, t_{j}\right] \cup\left[t_{j+1},+\infty\right), \forall j \in \mathbb{Z}$, and $v \in C_{0}^{\infty}\left(\left[t_{j}\right.\right.$, $\left.\left.t_{j+1}\right]\right)$. Hence, we have

$$
\begin{aligned}
0= & \int_{t_{j}}^{t_{j+1}}\left[|\dot{u}(t)|^{p-2}(\dot{u}(t), \dot{v}(t))+a(t)|u(t)|^{p-2}(u(t), v(t))\right] d t \\
& -\int_{t_{j}}^{t_{j+1}}(\nabla V(t, u(t)), v(t)) d t
\end{aligned}
$$

which implies that

$$
\frac{d}{d t}\left(|\dot{u}(t)|^{p-2} \dot{u}(t)\right)-a(t)|u(t)|^{p-2} u(t)+\nabla V(t, u(t))=0, \quad \text { a.e. } t \in\left(t_{j}, t_{j+1}\right) \text {. }
$$

The proof is complete.

\section{Proof of Theorem 1.1}

Proof of Theorem 1.1 Firstly, we prove that under the assumptions of Theorem 1.1, there exist $e \in E$ and $r>0$ such that $\|e\|>r$ and

$$
b:=\inf _{\|y\|=r} \varphi(y)>\varphi(0) \geq \varphi(e) .
$$

It is easy to see that $\varphi(0)=0$. From $(\mathrm{V} 2)^{\prime}$, there exists $\delta \in(0,1)$ such that

$$
|\nabla V(t, x)| \leq \frac{a_{0}}{2}|x|^{p-1} \quad \text { for } t \in \mathbb{R},|x| \leq \delta
$$

By $V(t, 0)=0$ and $(3.1)$, we have

$$
|V(t, x)| \leq \frac{a_{0}}{2 p}|x|^{p} \quad \text { for } t \in \mathbb{R},|x| \leq \delta
$$

Let $\|u\|=\frac{\delta}{C_{1}}:=r$, it follows from Lemma 2.3 that $|u(t)| \leq \delta$. From (2.6) and (3.2), we have

$$
\begin{aligned}
\varphi(u) & =\frac{1}{p}\|u\|^{p}+\sum_{j=-\infty}^{\infty} \int_{0}^{u\left(t_{j}\right)} I(t) d t-\int_{\mathbb{R}} V(t, u(t)) d t \\
& \geq \frac{1}{p}\|u\|^{p}-\frac{c m T^{p / q}}{p}\|\dot{u}\|_{L^{p}}^{p}-\frac{1}{2 p} \int_{\mathbb{R}} a_{0}|u(t)|^{p} d t
\end{aligned}
$$




$$
\begin{aligned}
& \geq \frac{1}{p}\|u\|^{p}-\frac{c m T^{p / q}}{p}\|\dot{u}\|_{L^{p}}^{p}-\frac{1}{2 p} \int_{\mathbb{R}} a(t)|u(t)|^{p} d t \\
& \geq \min \left\{\frac{1}{p}-\frac{c m T^{p / q}}{p}, \frac{1}{2 p}\right\}\|u\|^{p}:=b .
\end{aligned}
$$

Since $0<c<\frac{(\mu-p)}{(\mu+p) m T^{p / q}}$, we know that $b>0$. From Lemma 2.2(ii), we have for any $u \in E$

$$
\begin{aligned}
\int_{-3}^{3} V_{2}(t, u(t)) d t & =\int_{\{t \in[-3,3]:|u(t)|>1\}} V_{2}(t, u(t)) d t+\int_{\{t \in[-3,3]:|u(t)| \leq 1\}} V_{2}(t, u(t)) d t \\
& \leq \int_{\{t \in[-3,3]:|u(t)|>1\}} V_{2}\left(t, \frac{u(t)}{|u(t)|}\right)|u(t)|^{\varrho} d t+\int_{-3}^{3} \max _{|x| \leq 1} V_{2}(t, x) d t \\
& \leq\|u\|_{\infty}^{\varrho} \int_{-3}^{3} \max _{|x|=1} V_{2}(t, x) d t+\int_{-3}^{3} \max _{|x| \leq 1} V_{2}(t, x) d t \\
& \leq C_{1}^{\varrho}\|u\|^{\varrho} \int_{-3}^{3} \max _{|x|=1} V_{2}(t, x) d t+\int_{-3}^{3} \max _{|x| \leq 1} V_{2}(t, x) d t \\
& =C_{2}\|u\|^{\varrho}+C_{3},
\end{aligned}
$$

where $C_{2}=C_{1}^{\varrho} \int_{-3}^{3} \max _{|x|=1} V_{2}(t, x) d t, C_{3}=\int_{-3}^{3} \max _{|x| \leq 1} V_{2}(t, x) d t$. Take $\omega \in E$ such that

$$
|\omega(t)|= \begin{cases}1 & \text { for }|t| \leq 1 \\ 0 & \text { for }|t| \geq 3\end{cases}
$$

and $|\omega(t)| \leq 1$ for $|t| \in(1,3]$. For $s>1$, from Lemma 2.2(i) and (3.5), we get

$$
\int_{-1}^{1} V_{1}(t, s \omega(t)) d t \geq s^{\mu} \int_{-1}^{1} V_{1}(t, \omega(t)) d t=C_{4} s^{\mu}
$$

where $C_{4}=\int_{-1}^{1} V_{1}(t, \omega(t)) d t>0$. From (2.1), (2.6), (3.4), (3.5), (3.6), we get for $s>1$

$$
\begin{aligned}
\varphi(s \omega) & =\frac{s^{p}}{p}\|\omega\|^{p}+\sum_{j=-\infty}^{\infty} \int_{0}^{s \omega(t)} I(t) d t+\int_{\mathbb{R}}\left[V_{2}(t, s \omega(t))-V_{1}(t, s \omega(t))\right] d t \\
& \leq \frac{s^{p}}{p}\|\omega\|^{p}+\frac{c m T^{p / q} s^{p}}{p}\|\dot{\omega}\|_{L^{p}}^{p}+\int_{-3}^{3} V_{2}(t, s \omega(t)) d t-\int_{-1}^{1} V_{1}(t, s \omega(t)) d t \\
& \leq\left(\frac{1+c m T^{p / q}}{p}\right) s^{p}\|\omega\|^{p}+C_{2} s^{\varrho}\|\omega\|^{\varrho}+C_{3}-C_{4} s^{\mu} .
\end{aligned}
$$

Since $\mu>\varrho>p$ and $C_{4}>0$, it follows from (3.7) that there exists $s_{1}>1$ such that $\left\|s_{1} \omega\right\|>r$ and $\varphi\left(s_{1} \omega\right) \leq 0$. Set $e=s_{1} \omega(t)$, then $e \in E,\|e\|=\left\|s_{1} \omega\right\|>r$ and $\varphi(e)=\varphi\left(s_{1} \omega\right) \leq 0$.

Secondly, we prove that under the assumptions of Theorem 1.1, there exists a bounded sequence $\left\{u_{n}\right\}$ in $E$ such that

$$
\varphi\left(u_{n}\right) \rightarrow d, \quad \varphi^{\prime}\left(u_{n}\right) \rightarrow 0, \quad \operatorname{dist}\left(u_{n}, E\right) \rightarrow 0, \quad n \rightarrow \infty,
$$

where $\Phi=\{h \in C([0,1], E) \mid h(0)=0, h(1)=e\}, d=\inf _{h \in \Phi} \max _{s \in[0,1]} \varphi(h(s))$. Furthermore, $\left\{u_{n}\right\}$ does not converge to 0 in measure. 
From (I)' and Lemma 2.3, we have

$$
\sum_{j=-\infty}^{\infty}\left(I\left(u\left(t_{j}\right)\right), u\left(t_{j}\right)\right) \leq \sum_{j=-\infty}^{\infty}\left|I\left(u\left(t_{j}\right)\right)\right|\left|u\left(t_{j}\right)\right| \leq \sum_{j=-\infty}^{\infty} c\left|u\left(t_{j}\right)\right|^{p} \leq c m T^{p / q}\|\dot{u}\|_{L^{p}}^{p} .
$$

From (2.1), (2.2), (2.6), (3.9), (V3)', and (V5), we have

$$
\begin{aligned}
p d+\frac{p d}{\mu}\left\|u_{n}\right\| \geq & p \varphi\left(u_{n}\right)-\frac{p}{\mu}\left\langle\varphi^{\prime}\left(u_{n}\right), u_{n}\right\rangle \\
= & \frac{\mu-p}{\mu}\left\|u_{n}\right\|^{p}+p \sum_{j=-\infty}^{\infty} \int_{0}^{u\left(t_{j}\right)} I(s) d s-\frac{p}{\mu} \sum_{j=-\infty}^{\infty}\left(I\left(u_{n}\left(t_{j}\right)\right), u_{n}\left(t_{j}\right)\right) \\
& -p \int_{\mathbb{R}}\left[V_{1}\left(t, u_{n}(t)\right)-\frac{1}{\mu}\left(\nabla V_{1}\left(t, u_{n}(t)\right), u_{n}(t)\right)\right] d t \\
& +p \int_{\mathbb{R}}\left[V_{2}\left(t, u_{n}(t)\right)-\frac{1}{\mu}\left(\nabla V_{2}\left(t, u_{n}(t)\right), u_{n}(t)\right)\right] d t \\
\geq & \frac{\mu-p}{\mu}\left\|u_{n}\right\|^{p}-c m T^{p / q}\left\|\dot{u}_{n}\right\|_{L^{p}}^{p}-\frac{p c m T^{p / q}}{\mu}\left\|\dot{u}_{n}\right\|_{L^{p}}^{p} \\
\geq & \left(\frac{\mu-p}{\mu}-\frac{(\mu+p) c m T^{p / q}}{\mu}\right)\left\|u_{n}\right\|^{p} .
\end{aligned}
$$

Since $0<c<\frac{(\mu-p)}{(\mu+p) p T^{p / q}}$, the above inequalities implies that there exists a constant $C_{5}>0$ such that

$$
\left\|u_{n}\right\| \leq C_{5}, \quad n \in \mathbb{N} .
$$

By $(\mathrm{V} 2)^{\prime}$, we have

$$
\frac{1}{p}(\nabla V(t, x), x)-V(t, x)=o\left(|x|^{p}\right) \quad \text { as } x \rightarrow 0, t \in \mathbb{R}
$$

which implies that

$$
C_{6}:=\sup _{|x| \leq C_{1} C_{5}} \frac{\frac{1}{p}(\nabla V(t, x), x)-V(t, x)}{|x|^{p}}<\infty .
$$

For any $\varepsilon_{1}>0$, there exists $\delta_{1}>0$ such that

$$
\frac{1}{p}(\nabla V(t, x), x)-V(t, x) \leq \varepsilon_{1} a_{0}|x|^{p}, \quad|x| \leq \delta_{1}, t \in \mathbb{R} .
$$

It follows from (3.10), (3.12), and (3.13) that

$$
\begin{aligned}
\int_{\mathbb{R}} & {\left[\frac{1}{p}\left(\nabla V\left(t, u_{n}(t)\right), u_{n}(t)\right)-V\left(t, u_{n}(t)\right)\right] d t } \\
= & {\left[\int_{\left\{\mathbb{R}:\left|u_{n}(t)\right|>\delta_{1}\right\}}+\int_{\left\{\mathbb{R}:\left|u_{n}(t)\right| \leq \delta_{1}\right\}}\right]\left[\frac{1}{p}\left(\nabla V\left(t, u_{n}(t)\right), u_{n}(t)\right)-V\left(t, u_{n}(t)\right)\right] d t } \\
& \leq \int_{\left\{\mathbb{R}:\left|u_{n}(t)\right|>\delta_{1}\right\}} C_{6}\left|u_{n}(t)\right|^{p} d t+\int_{\left\{\mathbb{R}:\left|u_{n}(t)\right| \leq \delta_{1}\right\}} \varepsilon_{1} a_{0}\left|u_{n}(t)\right|^{p} d t
\end{aligned}
$$




$$
\begin{aligned}
& \leq \int_{\left\{\mathbb{R}:\left|u_{n}(t)\right|>\delta_{1}\right\}} C_{6}\left|u_{n}(t)\right|^{p} d t+\int_{\left\{\mathbb{R}:\left|u_{n}(t)\right| \leq \delta_{1}\right\}} \varepsilon_{1} a(t)\left|u_{n}(t)\right|^{p} d t \\
& \leq \operatorname{meas}\left\{\left|u_{n}(t)\right|>\delta_{1}\right\} C_{6}\left\|u_{n}\right\|_{\infty}^{p}+\varepsilon_{1}\left\|u_{n}\right\|^{p} \\
& \leq \operatorname{meas}\left\{\left|u_{n}(t)\right|>\delta_{1}\right\} C_{6} C_{1}^{p}\left\|u_{n}\right\|^{p}+\varepsilon_{1}\left\|u_{n}\right\|^{p} \\
& \leq \operatorname{meas}\left\{\left|u_{n}(t)\right|>\delta_{1}\right\} C_{5}^{p} C_{6} C_{1}^{p}+\varepsilon_{1} C_{5}^{p} .
\end{aligned}
$$

If $\left\{u_{n}\right\}$ converges to 0 in measure, from (I) and (3.14), we have

$$
\begin{aligned}
0< & d=\varphi\left(u_{n}\right)-\frac{1}{p}\left\langle\varphi^{\prime}\left(u_{n}\right), u_{n}\right\rangle+o(1) \\
= & \int_{\mathbb{R}}\left[\frac{1}{p}\left(\nabla V\left(t, u_{n}(t)\right), u_{n}(t)\right)-V\left(t, u_{n}(t)\right)\right] d t \\
& +\sum_{j=-\infty}^{\infty} \int_{0}^{u_{n}\left(t_{j}\right)} I(s) d s-\frac{1}{p} \sum_{j=-\infty}^{\infty}\left(I\left(u_{n}\left(t_{j}\right)\right), u_{n}\left(t_{j}\right)\right)+o(1) \\
\leq & \operatorname{meas}\left\{\left|u_{n}(t)\right|>\delta_{1}\right\} C_{5}^{p} C_{6} C_{1}^{p}+\varepsilon_{1} C_{5}^{p} \\
& +\frac{1}{p} \sum_{j=-\infty}^{\infty}\left[p \int_{0}^{u_{n}\left(t_{j}\right)} I(s) d s-\left(I\left(u_{n}\left(t_{j}\right)\right), u_{n}\left(t_{j}\right)\right)\right]+o(1) \\
= & o(1) .
\end{aligned}
$$

This is a contradiction. Hence, (3.8) holds and $\left\{u_{n}\right\}$ does not converge to 0 in measure.

Finally, from (3.10), we know that $u_{n} \rightarrow u$ in $E$, what we need to do is to prove that $\varphi^{\prime}(u)=0$. By (3.8), $\left\{u_{n}\right\}$ does not converge to 0 in measure and Lemma 2.4, there exists a sequence $\left\{x_{n_{k}}\right\}$ in $\mathbb{Z}$ such that $\omega_{k}:=u_{n_{k}}\left(\cdot+x_{n_{k}} T\right) \rightarrow u \neq 0$ in $E$. For any fixed $k \in \mathbb{N}$, set $s=t+x_{n_{k}} T$ and $v_{k}(s):=v\left(s-x_{n_{k}} T\right)$. Then $s_{j}:=t_{j}+x_{n_{k}} T(j \in \mathbb{Z})$ are impulsive points and

$$
\begin{aligned}
\left\|v_{k}\right\| & =\left\{\int_{\mathbb{R}}\left[\left|\dot{v}_{k}(s)\right|^{p}+a(s)\left|v_{k}(s)\right|^{p}\right] d s\right\}^{1 / p} \\
& =\left\{\int_{\mathbb{R}}\left[|\dot{v}(s)|^{p}+a(s)|v(s)|^{p}\right] d s\right\}^{1 / p}=\|v\| .
\end{aligned}
$$

For any $v \in C_{0}^{\infty}(\mathbb{R})$ with $v(k T)=0$, by $(\mathrm{V} 1)$ and (A), we have

$$
\begin{aligned}
\left\langle\varphi^{\prime}\left(\omega_{k}\right), v\right\rangle= & \int_{\mathbb{R}}\left[\left|\dot{\omega}_{k}(t)\right|^{p-2}\left(\dot{\omega}_{k}(t), \dot{v}(t)\right) d t+a(t)\left|\omega_{k}(t)\right|^{p-2}\left(\omega_{k}(t), v(t)\right)\right] d t \\
& -\int_{\mathbb{R}}\left(\nabla V\left(t, \omega_{k}(t)\right), v(t)\right) d t+\sum_{j=-\infty}^{\infty}\left(I\left(\omega_{k}\left(t_{j}\right)\right), v\left(t_{j}\right)\right) \\
= & \int_{\mathbb{R}}\left|\dot{u}_{n_{k}}\left(t+x_{n_{k}} T\right)\right|^{p-2}\left(\dot{u}_{n_{k}}\left(t+x_{n_{k}} T\right), \dot{v}(t)\right) d t \\
& +\int_{\mathbb{R}} a\left(s-x_{n_{k}} T\right)\left|\dot{u}_{n_{k}}\left(t+x_{n_{k}} T\right)\right|^{p-2}\left(u_{n_{k}}\left(t+x_{n_{k}} T\right), v(t)\right) d t \\
& -\int_{\mathbb{R}}\left(\nabla V\left(t, u_{n_{k}}\left(t+x_{n_{k}} T\right)\right), v(t)\right) d t+\sum_{j=-\infty}^{\infty}\left(I\left(u_{n_{k}}\left(t+x_{n_{k}} T\right)\right), v\left(t_{j}\right)\right) \\
= & \int_{\mathbb{R}}\left[\left|\dot{u}_{n_{k}}(s)\right|^{p-2}\left(\dot{u}_{n_{k}}(s), \dot{v}\left(s-x_{n_{k}} T\right)\right)\right.
\end{aligned}
$$




$$
\begin{aligned}
& \left.+a(s)\left|\dot{u}_{n_{k}}(s)\right|^{p-2}\left(u_{n_{k}}(s), v\left(s-x_{n_{k}} T\right)\right)\right] d t \\
& -\int_{\mathbb{R}}\left(\nabla V\left(s-x_{n_{k}} T, u_{n_{k}}(s)\right), v\left(s-x_{n_{k}} T\right)\right) d t \\
& +\sum_{j=-\infty}^{\infty}\left(I\left(u_{n_{k}}\left(s_{j}\right)\right), v\left(s_{j}-x_{n_{k}} T\right)\right) \\
= & \int_{\mathbb{R}}\left[\left|\dot{u}_{n_{k}}(s)\right|^{p-2}\left(\dot{u}_{n_{k}}(s), \dot{v}_{k}(s)\right)+a(s)\left|\dot{u}_{n_{k}}(s)\right|^{p-2}\left(u_{n_{k}}(s), v_{k}(s)\right)\right] d t \\
& -\int_{\mathbb{R}}\left(\nabla V\left(s, u_{n_{k}}(s)\right), v_{k}(s)\right) d t+\sum_{j=-\infty}^{\infty}\left(I\left(u_{n_{k}}\left(s_{j}\right)\right), v_{k}\left(s_{j}\right)\right) \\
= & \left\langle\varphi^{\prime}\left(u_{n_{k}}\right), v_{k}\right\rangle .
\end{aligned}
$$

By (3.17), we have

$$
\left|\left\langle\varphi^{\prime}\left(\omega_{k}\right), v\right\rangle\right|=\left|\left\langle\varphi^{\prime}\left(u_{n_{k}}\right), v_{k}\right\rangle\right| \leq\left\|\varphi^{\prime}\left(u_{n_{k}}\right)\right\|_{E^{*}} \cdot\left\|v_{k}\right\|=\left\|\varphi^{\prime}\left(u_{n_{k}}\right)\right\|_{E^{*}} \cdot\|v\|,
$$

where $E^{*}$ is the dual space of $E$. Equation (3.18) implies that

$$
\left\langle\varphi^{\prime}\left(\omega_{k}\right), v\right\rangle \rightarrow 0 \quad \text { as } k \rightarrow \infty
$$

For any $a>0$, let $\chi_{a}(t)=1$ for $t \in[-a, a]$ and $\chi_{a}(t)=0$ for $t \in(-\infty,-a) \cup(a, \infty)$. Then from (2.2), we have

$$
\begin{aligned}
& \left\langle\varphi^{\prime}\left(u_{n}\right)-\varphi^{\prime}(u), \chi_{a}\left(u_{n}-u\right)\right\rangle \\
& =\int_{-a}^{a}\left(\left|\dot{u}_{n}(t)\right|^{p-2} \dot{u}_{n}(t)-|\dot{u}(t)|^{p-2} \dot{u}(t), \dot{u}_{n}(t)-\dot{u}(t)\right) d t \\
& \quad+\int_{-a}^{a} a(t)\left(\left|u_{n}(t)\right|^{p-2} u_{n}(t)-|u(t)|^{p-2} u(t), u_{n}(t)-u(t)\right) d t \\
& \quad-\int_{-a}^{a}\left(\nabla V\left(t, u_{n}(t)\right)-\nabla V(t, u(t)), u_{n}(t)-u(t)\right) d t \\
& \quad+\sum_{t_{j} \in[-a, a]}\left(I\left(t, u_{n}\left(t_{j}\right)\right)-I\left(t, u\left(t_{j}\right)\right), u_{n}\left(t_{j}\right)-u\left(t_{j}\right)\right) \\
& \geq \int_{-a}^{a}\left|\dot{u}_{n}(t)-\dot{u}(t)\right|^{p} d t+\int_{-a}^{a} a(t)\left|u_{n}(t)-u(t)\right|^{p} d t \\
& \quad-\int_{-a}^{a}\left(\nabla V\left(t, u_{n}(t)\right)-\nabla V(t, u(t)), u_{n}(t)-u(t)\right) d t \\
& \quad+\sum_{t_{j} \in[-a, a]}\left(I\left(t, u_{n}\left(t_{j}\right)\right)-I\left(t, u\left(t_{j}\right)\right), u_{n}\left(t_{j}\right)-u\left(t_{j}\right)\right) .
\end{aligned}
$$

Since $\varphi^{\prime}\left(u_{n}\right) \rightarrow 0$ as $n \rightarrow+\infty$ and $u_{n} \rightarrow u$ in $E$, it follows from (3.8) that

$$
\begin{aligned}
& \left\langle\varphi^{\prime}\left(u_{n}\right)-\varphi^{\prime}(u), \chi_{a}\left(u_{n}-u\right)\right\rangle \rightarrow 0 \quad \text { as } n \rightarrow \infty, \\
& \int_{-a}^{a}\left(\nabla V\left(t, u_{n}(t)\right)-\nabla V(t, u(t)), u_{n}(t)-u(t)\right) d t \rightarrow 0 \quad \text { as } n \rightarrow \infty
\end{aligned}
$$


and

$$
\sum_{t_{j} \in[-a, a]}\left(I\left(t, u_{n}\left(t_{j}\right)\right)-I\left(t, u\left(t_{j}\right)\right), u_{n}\left(t_{j}\right)-u\left(t_{j}\right)\right) \rightarrow 0 \quad \text { as } n \rightarrow \infty
$$

It follows from (3.20), (3.21), (3.22), and (3.23) that

$$
\int_{-a}^{a}\left|\dot{u}_{n}(t)-\dot{u}(t)\right|^{p} d t \rightarrow 0, \quad \int_{-a}^{a} a(t)\left|u_{n}(t)-u(t)\right|^{p} d t \rightarrow 0 \quad \text { as } n \rightarrow \infty .
$$

Assume that for some $A>0, \operatorname{supp}(v) \subset[-A, A]$. Since

$$
\begin{aligned}
& \lim _{n \rightarrow \infty} \dot{u}_{n}(t)=\dot{u}(t), \quad \lim _{n \rightarrow \infty} u_{n}(t)=\dot{u}(t), \quad \forall \text { a.e. } t \in \mathbb{R}, \\
& \left|\left(\left|\dot{u}_{n}(t)\right|^{p-2} \dot{u}_{n}(t), \dot{v}(t)\right)\right| \leq \frac{p-1}{p}\left|\dot{u}_{n}(t)\right|^{p}+\frac{1}{p}|\dot{v}(t)|^{p}, \quad \forall t \in \mathbb{R}, n=1,2, \ldots, \\
& a(t)\left|\left(\left|u_{n}(t)\right|^{p-2} u_{n}(t), v(t)\right)\right| \\
& \leq a(t)\left[\frac{p-1}{p}\left|u_{n}(t)\right|^{p}+\frac{1}{p}|v(t)|^{p}\right], \quad \forall t \in \mathbb{R}, n=1,2, \ldots, \\
& \lim _{n \rightarrow \infty} \int_{-A}^{A}\left[\frac{p-1}{p}\left|\dot{u}_{n}(t)\right|^{p}+\frac{1}{p}|\dot{v}(t)|^{p}\right] d t \\
& =\frac{p-1}{p} \lim _{n \rightarrow \infty}\left\|\dot{u}_{n}\right\|_{L^{p}[-A, A]}^{p}+\frac{1}{p}\|\dot{v}\|_{L^{p}[-A, A]}^{p} \\
& =\frac{p-1}{p}\|\dot{u}\|_{L^{p}[-A, A]}^{p}+\frac{1}{p}\|\dot{v}\|_{L^{p}[-A, A]}^{p} \\
& =\int_{-A}^{A}\left[\frac{p-1}{p}|\dot{u}(t)|^{p}+\frac{1}{p}|\dot{v}(t)|^{p}\right] d t<+\infty
\end{aligned}
$$

and

$$
\begin{aligned}
\lim _{n \rightarrow \infty} \int_{-A}^{A} a(t)\left[\frac{p-1}{p}\left|u_{n}(t)\right|^{p}+\frac{1}{p}|v(t)|^{p}\right] d t \\
\quad=\frac{p-1}{p} \lim _{n \rightarrow \infty} \int_{-A}^{A} a(t)\left|u_{n}(t)\right|^{p} d t+\frac{1}{p} \int_{-A}^{A} a(t)|v(t)|^{p} d t \\
\quad=\frac{p-1}{p} \int_{-A}^{A} a(t)|u(t)|^{p} d t+\frac{1}{p} \int_{-A}^{A} a(t)|v(t)|^{p} d t<+\infty
\end{aligned}
$$

then it follows from (3.25), (3.26), (3.27), (3.28), (3.29), and the Lebesgue dominated convergence theorem that

$$
\int_{-A}^{A}\left(\left|\dot{u}_{n}(t)\right|^{p-2} \dot{u}_{n}(t), \dot{v}(t)\right) d t \rightarrow \int_{-A}^{A}\left(|\dot{u}(t)|^{p-2} \dot{u}(t), \dot{v}(t)\right) d t \quad \text { as } n \rightarrow \infty
$$

and

$$
\int_{-A}^{A} a(t)\left(\left|u_{n}(t)\right|^{p-2} u_{n}(t), v(t)\right) d t \rightarrow \int_{-A}^{A} a(t)\left(|u(t)|^{p-2} u(t), v(t)\right) d t \quad \text { as } n \rightarrow \infty
$$


For any $v \in E$ and $\varepsilon>0$, take $J_{0}$ sufficiently large such that

$$
\left(\sum_{j=J_{0}+1}^{+\infty}\left|v\left(t_{j}\right)\right|^{p}\right)^{1 / p} \leq \varepsilon, \quad\left(\sum_{j=-\infty}^{-J_{0}-1}\left|v\left(t_{j}\right)\right|^{p}\right)^{1 / p} \leq \varepsilon .
$$

Since $u_{n} \rightarrow u$ in $E, u_{n} \rightarrow u$ in $H^{1}\left(\left[t_{-J_{0}}, t_{J_{0}}\right]\right)$, therefore $u_{n}$ uniformly converges to $u$ in $\left[t_{-J_{0}}, t_{J_{0}}\right]$. By the continuity of $I$, there exists $N>0$ such that, for $n>N$, we have

$$
\left|\sum_{j=-J_{0}}^{J_{0}}\left[I\left(u_{n}\left(t_{j}\right)\right)-I\left(u\left(t_{j}\right)\right)\right] v\left(t_{j}\right)\right| \leq \varepsilon .
$$

From $(\mathrm{I})^{\prime}$, we have

$$
\begin{aligned}
& \left(\sum_{j=J_{0}+1}^{+\infty}\left[I\left(u_{n}\left(t_{j}\right)\right)-I\left(u\left(t_{j}\right)\right)\right]^{p /(p-1)}\right)^{(p-1) / p} \\
& \quad \leq\left(\sum_{j=J_{0}+1}^{+\infty}\left[c\left(\left|u_{n}\left(t_{j}\right)\right|^{p-1}+\left|u\left(t_{j}\right)\right|^{p-1}\right)\right]^{p /(p-1)}\right)^{(p-1) / p} \\
& \leq 2^{1 / p} c\left(\sum_{j=J_{0}+1}^{+\infty}\left(\left|u_{n}\left(t_{j}\right)\right|^{p}+\left|u\left(t_{j}\right)\right|^{p}\right)\right)^{(p-1) / p} \\
& \leq 2^{1 / p} c\left(m T^{p / q}\left\|\dot{u}_{n}\right\|_{L^{p}}^{p}+m T^{p / q}\|\dot{u}\|_{L^{p}}^{p}\right)^{1 / q} \\
& \leq 2 c m^{1 / q} T^{p / q^{2}} \max _{\left\{\sup _{n}\left\|\dot{u}_{n}\right\|_{L^{p}}^{p / q},\|\dot{u}\|_{L^{p}}^{p / q}\right\}}
\end{aligned}
$$

where $q=\frac{p}{p-1}$. Similarly, we have

$$
\begin{aligned}
& \left(\sum_{j=-J_{0}-1}^{-\infty}\left[I\left(u_{n}\left(t_{j}\right)\right)-I\left(u\left(t_{j}\right)\right)\right]^{p /(p-1)}\right)^{(p-1) / p} \\
& \leq 2 c m^{1 / q} T^{p / q^{2}} \max \left\{\sup _{n}\left\|\dot{u}_{n}\right\|_{L^{p}}^{p / q},\|\dot{u}\|_{L^{p}}^{p / q}\right\} .
\end{aligned}
$$

It follows from (3.33), (3.34), (3.35), and the Cauchy-Schwarz inequality that

$$
\begin{aligned}
\mid \sum_{j=-\infty}^{+\infty} & {\left[I\left(u_{n}\left(t_{j}\right)\right)-I\left(u\left(t_{j}\right)\right)\right] v\left(t_{j}\right) \mid } \\
\leq & \left|\sum_{j=J_{0}+1}^{+\infty}\left[I\left(u_{n}\left(t_{j}\right)\right)-I\left(u\left(t_{j}\right)\right)\right] v\left(t_{j}\right)\right| \\
& +\left|\sum_{j=-J_{0}}^{J_{0}}\left[I\left(u_{n}\left(t_{j}\right)\right)-I\left(u\left(t_{j}\right)\right)\right] v\left(t_{j}\right)\right| \\
& +\left|\sum_{j=-\infty}^{-J_{0}-1}\left[I\left(u_{n}\left(t_{j}\right)\right)-I\left(u\left(t_{j}\right)\right)\right] v\left(t_{j}\right)\right|
\end{aligned}
$$




$$
\begin{aligned}
\leq & \left(\sum_{j=J_{0}+1}^{+\infty}\left|I\left(u_{n}\left(t_{j}\right)\right)-I\left(u\left(t_{j}\right)\right)\right|^{q}\right)^{1 / q}\left(\sum_{j=J_{0}+1}^{+\infty}\left|v\left(t_{j}\right)\right|^{p}\right)^{1 / p} \\
& +\left|\sum_{j=-J_{0}}^{J_{0}}\left[I\left(u_{n}\left(t_{j}\right)\right)-I\left(u\left(t_{j}\right)\right)\right] v\left(t_{j}\right)\right| \\
& +\left(\sum_{j=-J_{0}-1}^{-\infty}\left|I\left(u_{n}\left(t_{j}\right)\right)-I\left(u\left(t_{j}\right)\right)\right|^{q}\right)^{1 / q}\left(\sum_{j=-J_{0}-1}^{-\infty}\left|v\left(t_{j}\right)\right|^{p}\right)^{1 / p} \\
\leq & \left(1+4 c m^{1 / q} T^{p / q^{2}} \max \left\{\sup _{n}\left\|\dot{u}_{n}\right\|_{L^{p}}^{p / q},\|\dot{u}\|_{L^{p}}^{p / q}\right\}\right) \varepsilon .
\end{aligned}
$$

It follows from (3.36) that

$$
\lim _{n \rightarrow \infty} \sum_{j=-\infty}^{+\infty}\left(I\left(u_{n}\left(t_{j}\right)\right), v\left(t_{j}\right)\right)=\sum_{j=-\infty}^{+\infty}\left(I\left(u\left(t_{j}\right)\right), v\left(t_{j}\right)\right) .
$$

Notice that

$$
\int_{-A}^{A}\left(\nabla V\left(t, u_{n}(t)\right), v(t)\right) d t \rightarrow \int_{-A}^{A}(\nabla V(t, u(t)), v(t)) d t \quad \text { as } n \rightarrow \infty .
$$

Hence, from (3.30), (3.31), (3.37), and (3.38), we have

$$
\left\langle\varphi^{\prime}(u), v\right\rangle=\lim _{n \rightarrow \infty}\left\langle\varphi^{\prime}\left(u_{n}\right), v\right\rangle=0 .
$$

Therefore, $\varphi^{\prime}(u)=0$ and $u$ is a nontrivial homoclinic solution of $\varphi$.

\section{Competing interests}

The authors declare that they have no competing interests.

\section{Authors' contributions}

All authors read and approved the final manuscript.

\section{Acknowledgements}

This work is supported by the Scientific Research Foundation of Guangxi Education Office of China (No. 2013LX171) and the Scientific Research Foundation of Guilin University of Aerospace Technology (No. YJ1301).

Received: 28 July 2014 Accepted: 16 September 2014 Published online: 02 October 2014

\section{References}

1. Fang, H, Duan, $\mathrm{H}$ : Existence of nontrivial weak homoclinic orbits for second-order impulsive differential equations. Bound. Value Probl. 2012, 138 (2012)

2. Bainov, DD, Simeonov, PS: Impulsive Differential Equations: Periodic Solutions and Applications. Longman, New York (1993)

3. Lakshmikantham, V, Bainov, DD, Simeonov, PS: Theory of Impulsive Differential Equations. World Scientific, Singapore (1989)

4. Chen, $\mathrm{P}$, Tang, $\mathrm{XH}$, Agarwal, RP: Fast homoclinic solutions for a class of damped vibration problems. Appl. Math. Comput. 219, 6053-6065 (2013)

5. Chen, P, Tang, XH: New existence and multiplicity of solutions for some Dirichlet problems with impulsive effects. Math. Comput. Model. 55, 723-739 (2012)

6. Chen, $\mathrm{H}$, Sun, J: An application of variational method to second-order impulsive differential equation on the half-line. Appl. Math. Comput. 217, 1863-1869 (2010)

7. Gong, W, Zhang, Q, Tang, XH: Existence of subharmonic solutions for a class of second-order p-Laplacian systems with impulsive effects. J. Appl. Math. 2012, Article ID 434938 (2012)

8. Luo, Z, Xiao, J, Xu J: Subharmonic solutions with prescribed minimal period for some second-order impulsive differential equations. Nonlinear Anal. 75, 2249-2255 (2012) 
9. Nieto, JJ, O'Regan, D: Variational approach to impulsive differential equations. Nonlinear Anal., Real World Appl. 10, 680-690 (2009)

10. Sun, J, Chen, $\mathrm{H}$ : Variational method to the impulsive equation with Neumann boundary conditions. Bound. Value Probl. 2009, Article ID 316812 (2009)

11. Sun, J, Chen, $H$, Yang, L: The existence and multiplicity of solutions for an impulsive differential equation with two parameters via a variational method. Nonlinear Anal. 73, 440-449 (2010)

12. Sun, J, Chen, H, Nieto, JJ, Otero-Novoa, M: The multiplicity of solutions for perturbed second-order Hamiltonian systems with impulsive effects. Nonlinear Anal. TMA 72, 4575-4586 (2010)

13. Sun, J, Chen, H, Nieto, JJ: Infinitely many solutions for second-order Hamiltonian system with impulsive effects. Math. Comput. Model. 54, 544-555 (2011)

14. Zhang, D, Dai, B: Existence of solutions for nonlinear impulsive differential equations with Dirichlet boundary conditions. Math. Comput. Model. 53, 1154-1161 (2011)

15. Zhang, Z, Yuan, R: An application of variational methods to Dirichlet boundary value problem with impulses. Nonlinear Anal., Real World Appl. 11, 155-162 (2010)

16. Zhou, J, Li, Y: Existence and multiplicity of solutions for some Dirichlet problems with impulsive effects. Nonlinear Anal. 71, 2856-2865 (2009)

17. Zhou, J, Li, Y: Existence of solutions for a class of second-order Hamiltonian systems with impulsive effects. Nonlinear Anal. TMA 72, 1594-1603 (2010)

18. Han, X, Zhang, H: Periodic and homoclinic solutions generated by impulses for asymptotically linear and sublinear Hamiltonian system. J. Comput. Appl. Math. 235, 1531-1541 (2011)

19. Zhang, H, Li, ZX: Periodic and homoclinic solutions generated by impulses. Nonlinear Anal., Real World Appl. 1, 39-51 (2011)

20. Zhang, QF: Existence and multiplicity of fast homoclinic solutions for a class of damped vibration problems with impulsive effects. Abstr. Appl. Anal. 2014, Article ID 960276 (2014)

21. Brezis, H, Nirenberg, L: Remarks on finding critical points. Commun. Pure Appl. Math. 64, 939-963 (1991)

22. Tang, XH, Lin, XY: Homoclinic solutions for a class of second-order Hamiltonian systems. J. Math. Anal. Appl. 354 539-549 (2009)

23. Tang, $\mathrm{XH}$, Lin, $\mathrm{XY}$ : Existence of infinitely many homoclinic orbits in Hamiltonian systems. Proc. R. Soc. Edinb., Sect. A, Math. 141, 1103-1119 (2011)

24. Lieb, EH: On the lowest eigenvalue of the Laplacian for the intersection of two domains. Invent. Math. 74, 441-448 (1983)

doi:10.1186/s13661-014-0220-5

Cite this article as: Li and Chen: Existence of homoclinic solutions for a class of second order $p$-Laplacian systems

with impulsive effects. Boundary Value Problems 2014 2014:220.

\section{Submit your manuscript to a SpringerOpen ${ }^{\circ}$ journal and benefit from:}

- Convenient online submission

Rigorous peer review

- Immediate publication on acceptance

- Open access: articles freely available online

- High visibility within the field

- Retaining the copyright to your article 